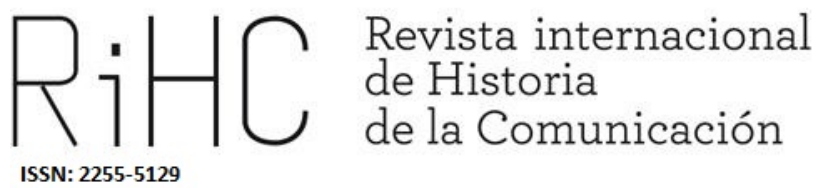

\title{
LA COMUNICACIÓN LOCAL EN LA TRANSICIÓN: TRANSPARENCIA Y PARTICIPACIÓN EN LAS JORNADAS DE INFORMACIÓN Y COMUNICACIÓN MUNICIPAL DE $1980^{1}$
}

Local media in the transition to democracy: transparency and participation in the Conference on Local Information and Communication in 1980

DOI: http://dx.doi.org/10.12795/RiHC.2018.i10.14

Recibido: 16/04/2018

Aceptado: $14 / 05 / 2018$

Publicado: $25 / 06 / 2018$

Pedro Molina Rodríguez-Navas (iD https://orcid.org/0000-0002-1586-881X

\footnotetext{
${ }^{1}$ Este artículo es resultado del proyecto de Investigación titulado Metodologías y modelos de información para el seguimiento de la acción de los responsables de los gobiernos locales y la rendición de cuentas, referencia CSO2015-64568-R, financiado por la Secretaria de Estado de Investigación, Desarrollo e Innovación, del Ministerio de Economía y Competitividad de España y el Fondo Europeo de Desarrollo Regional (FEDER), dentro del Programa Estatal de Investigación, Desarrollo e Innovación Orientada a los Retos de la Sociedad. Investigadoras principales Dra. Amparo Moreno Sardà y Dra. Núria Simelio Solà.
} 
Universidad Autónoma de Barcelona, pedro.molina@uab.cat

Resumen: Para estudiar el papel que tuvieron los medios de comunicación locales en España durante la transición a la democracia y las consecuencias de los modelos entonces establecidos, hay que conocer cuáles eran las cuestiones que consideraban relevantes los profesionales, políticos electos y académicos implicados en el área. Para ello, se estudian las ponencias presentadas en las Primeras Jornadas de Información y Comunicación Municipal, celebradas en Barcelona en 1980. En el análisis, se consideran tres campos de interés: la definición del contexto, la valoración de las cuestiones clave y la relevancia de los diferentes medios. La transparencia, la participación de la ciudadanía y la independencia de los profesionales, fueron considerados aspectos esenciales para conseguir una comunicación en beneficio de la comunidad local. A pesar de las radicales transformaciones que se han dado desde entonces, estos grandes problemas subsisten en los medios locales actuales.

Palabras clave: comunicación local, transición, democracia, participación, transparencia.

\begin{abstract}
To study the role of local media in Spain during the transition to democracy and the consequences of the models implemented since then, we must know what issues professionals, elected politicians and academics involved in the area considered relevant. For this reason, the papers presented at the First Conference on Local Information and Communication, held in Barcelona in 1980, have been studied. Three fields of interest are considered for the analysis: the definition of the context, the assessment of the key issues and the relevance of the different media. Transparency, citizen's participation and the independence of professionals were considered essential aspects to achieve communication for the benefit of the local community. In spite of the radical transformations that have taken place since then, these great problems persist in the current local media.
\end{abstract}

Keywords: local communication, transition, democracy, participation, transparency.

\title{
Introducción: objeto de estudio y alcance del problema
}

Los primeros gobiernos locales de la democracia en España se interesaron por encontrar la manera adecuada de comunicar a la ciudadanía las actuaciones que llevaban a cabo. Algunos autores han destacado que, tras la dictadura, la confluencia de objetivos de los diferentes partidos políticos facilitó la creación de gabinetes de prensa y de medios públicos (Corcoy et al., 2001).

En ese contexto se celebraron en el Palacio de Congresos de Montjuïc de Barcelona las Primeras Jornadas de Información y Comunicación Municipal, en las que participaron tanto profesionales de la comunicación como representantes políticos de 
ayuntamientos y académicos. Las ponencias y conclusiones de las Jornadas fueron publicadas en diferentes números de CEUMT. La Revista Municipal (1980a; 1980b; 1981) del Centro de Estudios Urbanísticos Municipales y Territoriales.

Las Jornadas se realizaron en un momento clave, ya que los primeros ayuntamientos democráticos se habían constituido un año y medio antes. En ese breve periodo de tiempo, profesionales y gobiernos municipales adquirieron una incipiente experiencia, desarrollaron interesantes trabajos y detectaron las dificultades con que topaban. Los autores se refieren a la importancia de esa etapa en muchas de las ponencias. Así, el texto de Moragas (1980: 9) comienza ubicando los problemas de la comunicación en España "a los dos años de aprobada la constitución" o Manuel Campo Vidal (1980: 20) sitúa los fenómenos que describe en una equidistancia entre las primeras elecciones y las siguientes.

El encuentro fue el marco adecuado en un momento de grandes expectativas no exento de incertidumbres. Junto a la presentación de experiencias, se expusieron diagnósticos sobre las carencias de los equipos de comunicación municipales y sobre los problemas de organización que era necesario abordar, constatándose también cuáles eran las aspiraciones y demandas que profesionales, ciudadanía y responsables políticos hacían a la comunicación local. También se aportaron propuestas y soluciones, reflexionando prioritariamente en torno a los medios de titularidad pública, aunque también sobre los medios privados de propiedad social o empresarial.

Participaron personalidades como los jefes de prensa de los ayuntamientos de Barcelona y Hospitalet de Llobregat, Josep Maria Soria y Jordi Negre respectivamente, periodistas como Huertas Clavería o Manuel Campo Vidal, representantes de medios de comunicación ciudadanos, del mundo académico, político, etc. El estudio de las aportaciones que hicieron a las Jornadas permite comprender cuál era la dirección en que se estaba trabajando, las preocupaciones fundamentales, los conceptos que se manejaban y las líneas maestras del contexto social y político, marcado todavía en buena parte por la herencia recibida de la dictadura. Este punto de partida permite valorar los éxitos, las dificultades y cambios de orientación que posteriormente se dieron en el cumplimiento de esos objetivos.

Es especialmente interesante comprobar como algunos de los considerados actualmente temas clave ya lo eran entonces, tales como la transparencia, la participación, la organización de los gabinetes municipales y la utilidad y efectividad de los diferentes medios. Fueron abordados partiendo de considerar que la comunicación debía contribuir al fortalecimiento de la democracia desde el ámbito local, cuestión que sigue siendo prioritaria (Molina, 2011), teniendo en cuenta diferentes ámbitos como el cultural o el político y social. No es ajena a esta cuestión la organización del evento en Barcelona, ya que la comunicación local y de proximidad tiene en Cataluña una larga tradición (Guillamet, 1983) y las reivindicaciones culturales, incluyendo el 
uso de la lengua catalana en los medios, han sido un tema fundamental tratado con cierta controversia (Guillamet 1983; Figueres 1983; Guimerà, 2004; Costa, 2009). También justifica el emplazamiento y el éxito de la convocatoria la concentración de grandes ciudades en el área de Barcelona, elemento determinante para el desarrollo de importantes áreas de comunicación en esos ayuntamientos.

Las precarias condiciones de trabajo no se resolvieron fácilmente, ni tampoco los medios públicos locales han dado cuenta después de la pluralidad política y social, a pesar de que las tecnologías han ido ofreciendo nuevas oportunidades (Corcoy et al. 2001; Costa et al. 2006; Costa, 2009), fenómeno compartido en el entorno europeo (Cavadas, 2012). Desde el inicio de la democracia se puso de manifiesto la tensión entre el ideal de comunicación pública contribuyendo al desarrollo de mejores formas democráticas y la utilización de los medios por parte de los gobiernos municipales para perpetuarse en el poder.

Esta tensión condujo a la creciente importancia de los medios empresariales sobre los vecinales y sociales que tanto habían aportado en aquellos años iniciales (Maristany y Musons 2002; Costa, 2009), a la subsidiaria presencia de las televisiones municipales en la actualidad (Guimerà y Alborch, 2011) y la drástica reducción de lo que Guillamet (1983) llamó el "periodismo popular". Aunque los actuales medios digitales parecen abrir campos para la participación y las transformaciones sociales (Castells, 2009) sus posibilidades y características, si bien entroncan con las propuestas de Enzensberger (1972) o MacBride (1980) en boga entonces, han configurado nuevas formas de relación-comunicación en un contexto global drásticamente diferente al de 1980. La perspectiva actual sitúa el problema en el diseño de mecanismos de dación de cuentas (Romero y Farinós, 2011), sin ocultar que algunas de las prácticas son más bien utilizadas para amortiguar conflictos (Martínez y Rosende, 2011; Rojo, 2005; Jessop 2003; Peters, 2000).

Marta Corcoy (2016) ha analizado las publicaciones municipales en papel de una muestra de municipios de Catalunya entre 1979-2009 y no aprecia grandes cambios en todo el periodo, afirmando que no se ha fomentado el debate ciudadano. En ese mismo trabajo, analiza el proceso de implantación de las TIC en los muncipios hasta 2015 y su repercusión en las informaciones. El resultado es que la utilización de estos recursos tecnológicos no ha servido para promover la participación, ni han conllevado por si solos un cambio en la calidad de las informaciones, que continúan estando al servicio de intereses partidistas, sin que se utilicen para hacer balance de la gestión o rendimiento de cuentas.

Posteriormente, el seguimiento de la publicación de noticias en las web municipales hecho por Manfredi, Corcoy y Heerranz (2017) entre 2011 y 2016 demuestra que ha aumentado la cantidad pero no la calidad. Estos mismos autores ponen de manifiesto la falta de interés que se percibe por mejorar esa situación, destacando que ni el color 
político ni el género del alcalde o alcaldesa pueden relacionarse con prácticas mejores o peores: los miembros de la oposición continúan sin tener la presencia que les corresponde como representantes de la ciudadanía, no se publican las crónicas de los plenos municipales en la mayor parte de las webs corporativas, ni se publican informaciones suficientes sobre mecanismos de participación. Sobre este último punto, Catalina y García (2013), concluyen afirmando que ni los ayuntamientos tienen como finalidad propiciar una relación con la ciudadanía, ni la voluntad de hacerlo tiene presente una relación directa con la magnitud poblacional del municipio estudiado. A conclusiones semejantes apuntan los estudios sobre la rendición de cuentas, señalando que la incorporación de nuevos instrumentos digitales no implica un cambio en los comportamientos, en la mayor parte de los casos (Rivero, Mora y Flores, 2007).

Finalmente, otra cuestión de plena actualidad es la transparencia, ya que la Ley de Transparencia estatal fue aprobada en $2013^{2}$ y posteriormente han sido sancionadas otras de ámbito autónomico, como la de Cataluña ${ }^{3}$. El estudio de las ponencias presentadas en las Jornadas revela la gran importancia que ya se le otorgaba en 1980, más relacionada con el derecho a la información y la participación que con la prevención de la corrupción, tal y como también se plantea hoy (Bertot, Jaeger y Grims, 2010). Pero, a pesar de esto, y aunque las investigaciones han ido demostrando reiteradamente la necesidad de contar con legislación para amparar el derecho de acceso a la información para impulsar un cambio en los comportamientos y actitudes de los gobiernos municipales (Moreno et al. 2013), la legislación vigente en España no ha sido aprobada hasta que la corrupción se ha convertido en un grave problema que socaba la credibilidad política y cuestiona las instituciones democráticas (Gértrudix, et al., 2016). Sin embargo, en el redactado de las leyes se concede relevancia principal a la participación política de la ciudadanía.

Con todo, los estudios demuestran que 38 años más tarde, la implantación de estas leyes no se ha completado en el ámbito municipal, a pesar de su plena vigencia desde finales de 2015. Los trabajos más recientes (Molina et al., 2017; Moreno, Molina y Simelio, 2017) demuestran que la legislación sobre transparencia no ha tenido un impacto suficiente, ni tan solo en los municipios mayores, considerando tanto criterios de cantidad como de calidad de la información.

\footnotetext{
${ }^{2}$ Ley 19/2013, de 9 de diciembre, de transparencia, acceso a la información pública y buen gobierno. (BOE núm. 18, de 21 de enero de 2015), BOE, en https://www.boe.es/buscar/doc.php?id=BOE-A-2015470.

${ }^{3}$ Ley 19/2014, de 29 de diciembre, de transparencia, acceso a la información pública y buen gobierno. Comunidad Autónoma de Cataluña. (BOE núm. 18, de 21 de enero de 2015), BOE, en http://www.boe.es/buscar/act.php?id=BOE-A-2015-470
} 


\section{Objetivos, metodología y fuentes}

\subsection{Objetivos, hipótesis y metodología}

El objetivo de este trabajo es conocer cuáles eran las cuestiones que los profesionales de la comunicación, políticos electos y académicos, consideraban relevantes para que la comunicación local contribuyese al fortalecimiento de la democracia y de los ayuntamientos democráticos en el primer mandato municipal de la democracia.

Consideramos que se trata de un momento especialmente significativo, fundacional, y que la perspectiva y las soluciones que se adoptaron en aquel momento condicionaron la actividad desarrollada en el campo de la comunicación hasta la actualidad.

A pesar de esto, partimos de la hipótesis de considerar que los problemas que se detectaron en aquel momento perviven en la actualidad y que, por tanto, la comunicación pública local padece hoy problemas semejantes. Para verificarla, vamos a interrogar a las fuentes sobre tres cuestiones relativas a la función de la comunicación local en democracia: cuál era el contexto social, político y mediático; cómo se abordaban los asuntos esenciales: la transparencia, la participación de la ciudadanía y la organización de los servicios de comunicación municipales; y, por último, cuál era la función de los diferentes medios disponibles.

\subsection{Fuentes}

Para alcanzar el objetivo y validar la hipótesis, se han estudiado las ponencias presentadas en las Primeras Jornadas de Información y Comunicación Municipal de 1980. Para consultar estos documentos y calibrar la importancia de este acontecimiento, hemos hecho un trabajo de documentación.

Las Jornadas se celebraron en Barcelona entre los días 14, 15 y 16 de octubre de 1980. Asistieron 180 personas entre profesionales de la información, concejales y técnicos de ayuntamientos y diputaciones de toda España (CEUMT, 1980a: 8). Prueba del interés que suscitaron es el seguimiento que hizo el diario La Vanguardia (1980a, b y c) y la información aparecida en el $A B C$ de Sevilla, el domingo 19 de octubre, en la que se puede leer:

Han regresado de Barcelona, donde asistieron a las Primeras Jornadas de Información y Comunicación Municipal, organizadas por el CEUMT, el capitular Miguel Ángel González de la Puente (PSA), y el jefe del Gabinete de Prensa, José Luís de Vicente. Capitular y técnico han mantenido importantes reuniones con 
los representantes del resto de las capitales españolas que asistieron a los debates (ABC, 1980: 24).

Fueron organizadas por el Centre d'Estudis Urbanístics Municipals i Territorials, con sede en Barcelona. La publicación de las ponencias se hizo en la revista de la propia entidad, CEUMT. La Revista Municipal, aunque no de forma completa, como explicaremos más adelante. La revista contaba en ese momento con una edición de distribución estatal, en castellano, y otra edición para Cataluña en la que se añadían unas páginas en catalán con numeración independiente. Aunque para este trabajo hemos consultado la edición catalana, los textos de las Jornadas se publicaron en castellano en la edición nacional, concretamente en la sección Administración Local de cada uno de los números donde aparecieron.

En algunos de los trabajos se hacen referencias explicitas a asistentes a las Jornadas. Así, por ejemplo, en el texto de Jordi Negre (1980: 9) se alude a la presencia como invitada de Carmen Sánchez Larramburo, Jefa de Prensa del Ayuntamiento de Sant Cugat, entre otros. Igualmente, hay referencias a contenidos de intervenciones previas, por lo que presumimos que los redactados finales publicados se hicieron a partir de las grabaciones y posteriores transcripciones de las ponencias presentadas, tal y como parece indicarlo la nota introductoria en la revista a la tercera parte de las publicaciones, cuando se dice que los textos "han sido revisados por los propios autores" (CEUMT, 1981: 5). Incluso, en una de las ponencias se dice que entre la conferencia y el escrito hay grandes diferencias porque el autor no consideró conveniente "pulir la transcripción", prefiriendo hacer un texto más adecuado a una presentación escrita (Trias-Folch, 1981: 17).

Se publicaron 14 textos en los números 32, 33 y 35 de la revista, en un orden que no se corresponde con el de las intervenciones durante las Jornadas. Tampoco se imprimieron todas las ponencias (entre algunos de los textos se insertaron notas indicando cuales fueron los trabajos presentados, pero no publicados) ni todos los trabajos publicados eran ponencias completas, tal y como se advierte en los títulos.

Las ponencias publicadas (cuyas referencias constan en la bibliografía) fueron dictadas por Jordi Negre i Rigol, jefe de prensa del Ayuntamiento de Hospitalet de Llobregat; Josep Maria Soria, jefe de prensa del Ayuntamiento de Barcelona; Josep Maria Huertas Clavería, periodista; Manuel Campo Vidal, subdirector del diario Tele/eXpres; Miquel de Moragas, profesor de la Facultad de Ciencias de la Información (actualmente de la Comunicación) de la UAB; Manuel Vázquez Montalbán, quien también fue profesor en la Facultad de Ciencias de la Información de la UAB; Francisco Montalvo, publicitario; Anna Segura, publicitaria, y Ferran Cartes, diseñador gráfico y profesor de Teoría de la Comunicación en la Escola Massana de Barcelona; Michel Destot, concejal responsable de Información del Ayuntamiento de Grenoble; Joan Castelló Rovira, Jefe de Programación de Radio Barcelona; Pere Ignasi Fages, periodista; Jordi Antón González 
y Roser Carvajal, miembros del Institut del Cinema Català; Toni Garriga, miembro de la Comisión Gestora de RTV Cardedeu; y Josep María Trías Folch, presidente de la Asociación de Diseñadores Profesionales (ADP).

Se completó la participación extranjera con la intervención no publicada, de Nuno Portas, arquitecto y urbanista portugués, director de la revista Cadernos Municipais, que suplió la ponencia de Diego Novelli, alcalde de Turín, quien no pudo asistir (CEUMT, 1981: 5) pero que "envió un trabajo que fue recogido en las lecturas" (La Vanguardia, 1980c).

Tras la ponencia de Joan Castelló se presentaron dos comunicaciones no publicadas, pronunciadas por Antoni Esteve, Concejal de Información del Ayuntamiento de Arenys de Mar, y por Eduard Delgado, especialista en temas de animación cultural del Área de Descentralización y Participación del Ayuntamiento de Barcelona (CEUMT, 1981: 8).

También se pronunciaron dos ponencias más sobre vídeo, no publicadas: "a cargo de Jordi Parés, de VIDESPOT (...), y de Carles Ametller y Pau Margall de SERVEI DE VIDEO COMUNITARI" (CEUMT, 1981: 13).

Además, en el número 32 de CEMUT (1980a) se publicaron las conclusiones referentes a Organización de la Comunicación y la Información de los Ayuntamientos. Gracias a las informaciones aparecidas en La Vanguardia (1980c) sabemos de la existencia de dos apartados de conclusiones más, uno sobre radio y otro sobre prensa y materiales impresos, que no fueron publicados.

También por las informaciones del diario La Vanguardia (1980b), sabemos que el acto inaugural se desarrolló en el Saló de Cent del Ayuntamiento de Barcelona y que consistió en una bienvenida del Alcalde Accidental de Barcelona, Antoni Comes (en sustitución del alcalde Narcís Serra) y en el que dictaron sus ponencias Miquel de Moragas y Manuel Vázquez Montalbán.

La información del día 16 en La Vanguardia (1980a) anuncia una mesa redonda sobre Prensa y Política Municipal, que posteriormente fue reseñada en la información del día 18 (La Vanguardia, 1980c). Intervinieron el propio director de La Vanguardia, Horacio Sáenz Guerrero, Jesús de la Serna, en representación de El País, Adolfo Luxán (concejal del Ayuntamiento de Madrid) y Josep M. Soria, con la moderación de Josep Pernau.

En cuanto a la jornada de clausura (La Vanguardia, 1980c), además de algunas ponencias que posteriormente aparecieron publicadas, también intervinieron representantes de ayuntamientos como Hospitalet de Llobregat, Malaga, Sevilla y Valencia, entre otras que no se citan en el artículo fuente. También estaba prevista una exposición de "experiencias comunicativas en las distintas áreas de la gestión municipal", incluyendo la proyección de cortos del Noticiari Català, realizados por el 
Institut del Cinema Català y otros de "«Vídeo comunitario» sobre Santa Coloma de Gramenet, y de la TV de Cardedeu" (La Vanguardia, 1980c).

\section{Análisis de los textos}

\subsection{La comunicación local en el contexto nacional e internacional}

Las ponencias impartidas por académicos subrayaron la negativa permanencia de la herencia del franquismo y basaron sus propuestas de cambio en algunas de las más influyentes corrientes de pensamiento de la época.

Así, Miquel de Moragas calificaba el sistema comunicativo del momento de antidemocrático, porque: "No es suficiente garantizar el derecho formal a la libertad informativa, la democracia debe garantizar su realización práctica" (Moragas, 1980: 10) y en España no apreciaba la existencia de una política de comunicación. Por su parte, Manuel Vázquez Montalbán consideraba que los gobiernos progresistas no contaban con suficientes medios y citó a Giovanni Amándola para señalar la "acumulación histórica de reaccionarismo" (Vázquez Montalbán, 1980: 16) como barrera a la confianza que las políticas de las izquierdas no estaban rompiendo, ya que la gestión seguía modelos con escasa participación.

Montalbán describió en su conferencia el modelo que Hans Magnus Enzensberger (1972) había definido en Elementos para una teoría de los medios de comunicación y apuntó que: "Las ciudades son microsomas sociales que reproducen a escala planteamientos universales $\mathrm{y}$ en ellas pueden ensayarse revisiones culturales trascendentales" (Vázquez Montalbán, 1980: 17). También confiaba en los medios no masivos y en las "células sociales de base, desde el colegio hasta la fábrica" para avanzar hacia el "socialismo en libertad" (Ibid: 17-18).

Para llegar a conclusiones semejantes, Moragas se basó en el entonces reciente informe MacBride (1980) y en los resultados de otras conferencias internacionales, trazando cuál debía ser el papel de la comunicación local en relación al resto de los diferentes niveles. En su ponencia afirma:

La comunicación descentralizada, gestionada desde la institución municipal, o desde las asociaciones de base, se convierte, entonces en uno de los principales objetivos de la lucha por la democracia, amenazada de manera sutil, incluso con argumentos relativos a su propia defensa, desde la lógica desmovilizadora de la 
política entendida como un espectáculo, al que solo se accede a través de la participación ficticia que ofrecen los medios de comunicación de masas (Moragas, 1980: 15).

Moragas se refiere con cierta vehemencia a los desequilibrios del sistema comunicativo español, que impedían que las zonas que llama "subdesarrolladas y dependientes" (Ibid: 11) o las "históricamente reprimidas" (Ibid: 13) dispusieran de medios para suministrar su imagen. No solamente negaba la existencia de voluntad por promover la participación, sino que vislumbraba un empeoramiento de la situación con la aparición de televisiones privadas, interrogándose sobre las implicaciones que tendría para la prensa y el conjunto del sistema comunicativo catalán la existencia de televisión autonómica. Como contrapartida, y para promover la participación y la pluralidad, Moragas aprecia la "resistencia social que se expresa a través de los medios descentralizados" (Ibid: 14) y aboga por "la recuperación y una defensa intransigente de las costumbres comunicativas" (Ibid: 15$)$ en que el ámbito local debía tener una gran incidencia.

Josep Maria Soria aportó otro enfoque, más próximo al campo profesional, partiendo de los consejos elaborados en la Constitución del Comité Mundial de Periodistas de Información Local en el marco de la II Conferencia Mundial Intermunicipal de Fez (1979), que señalaron la necesidad de una información-comunicación entre ciudadanos y políticos, así como la de explicar las decisiones políticas publicando los documentos en que se fundamentan, de forma adaptada a las diferentes situaciones (Soria, 1980:14). En cuanto a la situación interna, Soria explicó el éxito de participación en las Jornadas como constatación de que los ayuntamientos no conseguían informar adecuadamente por la inexperiencia. También aprecia otros motivos, como la falta de determinación de algunos políticos y la escasa utilización de instrumentos como la publicidad y las relaciones públicas, que debían contribuir a paliar las deficiencias de los medios clásicos.

Compartió el diagnóstico el periodista Huertas Clavería (1980), quien expuso el caso catalán como ejemplo de que la práctica de la comunicación municipal no respondía a las expectativas generadas tras las primeras elecciones de la democracia ya que en la mayoría de las poblaciones no se habían creado oficinas de prensa, ni las existentes funcionaban a pleno rendimiento y, en cualquier caso, afirma que no se sabía cómo organizarlas satisfactoriamente. Señaló que durante la campaña electoral los partidos de izquierdas (aludiendo a PSC y PSUC) insistían en la necesidad de la transparencia, de la información exhaustiva sobre la gestión, pero puso de relieve la diferencia entre los principios y su aplicación en el ejercicio del poder. Contundentemente, lo expresaba de esta manera:

Conclusión evidente a priori, pero que conviene remachar, es pues que a la derecha -hoy rebautizada como centro o partido interclasista- no le va la 
información, prefiere oscuridad y manos quietas a luz y taquígrafos. Entre otras razones porque parte de sus elementos activos a nivel municipal provienen de antiguos consistorios franquistas, tras el correspondiente lavado democrático.

Otra conclusión, más delicada, pero que se puede también avanzar, es la de que a la izquierda la información le va por principios pero la teme en la práctica concreta, de cada día (Ibíd: 15).

A pesar de esto, recuerda que la situación de partida era precaria, que no se asignaban presupuestos para esas tareas, acusando a la UCD de boicotear los presupuestos municipales y a los partidos de la derecha en general (CDC y UCD) de obstaculizar la creación de los gabinetes de información municipales. A pesar de todo, reconoce la existencia de experiencias positivas, ya que aproximadamente 12 municipios de más de 20.000 habitantes contaban con oficina de prensa, aunque a veces se limitaban a la contratación de un profesional. Las dificultades con las que el periodista se topaba en esas circunstancias las ejemplifica aludiendo al caso real del "autosecuestro" (Ibid: 16) de un boletín municipal. La excepción a ese abandono, según Huertas Clavería, era la ciudad de Barcelona, ya que la izquierda utilizaba el ejemplo de la capital de Cataluña como "caja de resonancia" (Ibíd: 16) y por otros motivos relacionados con la importancia de la ciudad y el centralismo informativo.

También Jordi Negre (1980) constató la intensa dinámica que se había iniciado desde diferentes sectores relacionados con la comunicación local, exponiendo que paralelamente se celebraron las Primeras Jornadas de Periodistas Jefes de Gabinetes Municipales de Medios de Comunicación de las que ya se había realizado una primera reunión, en Hospitalet de Llobregat (3/7/1980); en la siguiente, que se celebraría en Manresa (12/11/1980), se debían discutir y aprobar las ponencias que en ese momento se estaban elaborando.

Dada la necesidad de contar con modelos, se presentaron experiencias de otros países europeos. Michel Destot (1981), concejal del Ayuntamiento de Grenoble, explicó la experiencia de esa ciudad, que según se dice en la introducción a la publicación: "Ha sido durante muchos años, y lo es, punto de referencia fundamental para toda experiencia de información y de comunicación municipal” (CEUMT, 1981: 5). El autor enfatiza el contraste entre las escasas oportunidades para la comunicación local en una Francia centralista y las actuaciones llevadas a cabo en Grenoble, resaltando que se destinaba el 0,5\% del presupuesto municipal a la comunicación, por lo que se podía contar con 12 personas para el servicio, incluyendo "periodistas-corresponsales, grafistas-montadores, azafatas de información en relación directa con el público" (Op. Cit.: 6). Además de referirse a la utilización de los medios de comunicación, Destot da mucha importancia a la comunicación directa e interna, por lo que resulta muy interesante la existencia de un boletín de información del personal, de publicaciones especializadas dirigidas a las asociaciones, de lo que llama "puntos de opinión" (Ibid: 
7), de reuniones informativas y, por último, de un proyecto en preparación de utilización de la telemática en 50 centros públicos, integrando teléfono, televisión y ordenador, para conseguir una mayor eficiencia "en beneficio de la democracia local" (Ibid: 7).

\subsection{Transparencia, participación y organización de los servicios de comunicación}

Transparencia y participación eran preocupaciones no solo relacionadas con el contexto propio de la transición, también se enmarcaban en un movimiento internacional que era conocido y utilizado en la argumentación, como ya hemos podido ver en el apartado anterior. Fueron dos temas clave, señalados en un buen número de ponencias como estrategias imprescindibles para los nuevos ayuntamientos democráticos. La voluntad de orientar la comunicación municipal hacia esos dos aspectos hacía imprescindible modificar la organización de los servicios.

En la segunda parte de su ponencia, Jordi Negre (1980) abordó la que consideraba sincera preocupación de los gobiernos municipales: ofrecer información transparente y buscar la participación de la ciudadanía. Argumentaba que la falta de criterios y de organización dificultaba que se pudiera llevar a cabo esa tarea, que debía partir de un Gabinete de Medios de Comunicación, dirigido por un periodista, y de un servicio de relaciones ciudadanas, dirigido por un especialista en comunicaciones públicas, ambos bajo la dirección de un político.

Incluso en un texto de cariz más técnico, Campañas de concienciación, de Anna Segura y Ferran Cartes (1980) y que según La Vanguardia (1980c), fue la presentación más debatida, la cuestión de la participación resulta central. En primer lugar, definieron como "Campañas de Concienciación a todas las acciones encaminadas a conseguir la toma de posición de un grupo humano" (Op. Cit.: 23), diferenciando tres tipos de toma de posición: aquiescencia, solidaridad y participación. Para alcanzar ese último estadio proponían la elaboración de un "Plan de Comunicación Permanente" (Ibid: 24) centrado en las características de la población para fijar el tono de las comunicaciones, detectando la evolución de los estados de opinión. También propusieron dotar a los ayuntamientos de una "Imagen de Empresa" (Ibid: 25), aunque todos los esfuerzos comunicativos de un ayuntamiento democrático se han de dirigir a explicar su actividad y a conseguir la solidaridad con sus actuaciones y la participación.

Huertas Clavería también dedicó un apartado de su ponencia a explicar las características que debía tener un gabinete de información municipal, como ausencia de sectarismo, información completa sin ocultar los asuntos delicados, creador de imagen especialmente centrada en la figura del alcalde y dirección profesional bajo 
supervisión política sin que esto signifique pérdida de independencia. Algunos de estos puntos resultan difícilmente compatibles, como cuando dice que los gabinetes deben "utilizar la figura del alcalde cara a consolidar sus cualidades, procurando limar asperezas que se hayan producido, errores cometidos o disipar el aire de figura inaccesible de que a menudo se rodean algunos alcaldes" (Huertas, 1980: 17). Tampoco parece muy acorde ejemplificar la independencia de los profesionales con asuntos ajenos a los contenidos como el tamaño del boletín o considerar inadecuado cubrir esas plazas mediante concursos ya que se trata de un profesional "supeditado a la política" (Ibid: 18).

Mientras Huertas Clavería sitúa el ámbito político e institucional en primer lugar, Manuel Campo Vidal atiende preferentemente a las necesidades de la ciudadanía, pues los gobiernos de izquierdas precisan de la población para hacer frente a otros poderes. Para enderezar esa situación, apunta que la información municipal es un "elemento de formación del ciudadano" (Ibid: 20) y que la prensa escrita tiene un papel fundamental para contrarrestar las informaciones recibidas por otros medios. Constata que en un primer momento los boletines municipales fueron excesivamente burocráticos, triunfalistas o pesimistas, incurriendo frecuentemente en el proselitismo y que no contaban con profesionales para su elaboración, pero que en una segunda fase mejoraron en muchos aspectos. El autor plantea ocho preguntas a las que políticos y técnicos municipales debieran dar respuesta para mejorar las condiciones de sus publicaciones, sobre los niveles de formación y la participación de la población, la acogida y utilidad de la publicación, el tipo de información que se ofrece e incluso reflexiona sobre la posibilidad de que ofrezcan servicios como los relacionados con la búsqueda de empleo. Sin embargo, declara que se trata de "una operación 'parcheo'" (Ibid: 23), ya que considera que en cada población debiera existir prensa independiente y que los ayuntamientos deberían apoyar su aparición e incluir en ella la información municipal.

En las conclusiones publicadas sobre Organización de la Comunicación y la Información de los Ayuntamientos (CEUMT, 1980a: 8) se aborda la cuestión de la transparencia informativa como un derecho de la población que "coincide con la voluntad política de los ayuntamientos democráticos" y se insta a profundizar invitando a todos los ayuntamientos a formar un área dirigida por un concejal, que debe "asegurar" esa transparencia. Para ello, se considera preciso dotarla de los recursos técnicos necesarios, poniendo de manifiesto la intención práctica de las Jornadas. Fueron un lugar de encuentro para compartir experiencias y dificultades con la voluntad de encontrar soluciones comunes. En conjunto, se aprecia en esas conclusiones un verdadero interés por fomentar una comunicación que dé cuenta de la acción del gobierno municipal, por recoger los intereses de los ciudadanos, ("vecinos" es el término utilizado) y por que los municipios dispusiesen de una organización adecuada y eficiente. A pesar de esto, también hubo ponencias, como la del publicitario 
Francisco Montalvo (1980), enfocadas desde el interés por conseguir la mayor eficacia de la administración.

\subsection{Diversidad de medios}

Otro asunto ampliamente tratado fue la utilidad de los diversos medios disponibles, no solamente los de titularidad pública, sino también la relación que debía establecerse con los medios privados. En este sentido, Soria (1980: 14) aludía a la necesidad de que las relaciones entre políticos y periodistas fueran fluidas, de forma que los periodistas pudieran contribuir para que la "democracia de participación estimule y vivifique la democracia de representación". Huertas Clavería comparte la misma opinión y se refiere a las relaciones con los medios privados de forma muy gráfica al señalar la necesidad de "mantener lubrificados los contactos" (Huertas, 1980: 19) para fomentar la presencia de los cargos electos en los mismos, proponiendo preparar para ello dossiers sobre diferentes temas y contactos periódicos del alcalde con la prensa.

Sobre esta cuestión, Negre (1980: 10) recoge que la prensa generalista se ocupa poco de las informaciones municipales o comarcales, siendo principalmente noticia cuando se dan "conflictos internos en el seno de los Ayuntamientos progresistas". Por este motivo, considera conveniente que se hagan esfuerzos por acercarse a los equipos directivos de la prensa y cree que las causas son la falta de criterios de los redactores de Barcelona para evaluar la importancia de las noticias en otras poblaciones y la "mediatización ideológica o política" de los corresponsales (Ibid: 10). Incluso resalta la gran importancia de las agencias informativas para los ayuntamientos, ya que los periódicos atienden con más interés a las noticias de las agencias que a las de sus propios corresponsales, porque les conceden mayor credibilidad. También critica que el monopolio estatal de televisión fuera especialmente inaccesible a los ayuntamientos gobernados por la izquierda y la insuficiente emisión de información sobre Cataluña, por lo que incita a los ayuntamientos a exigir un canal de televisión autonómico. Por el contrario, la radio le parece un medio idóneo, en auge y sin acusada decantación política.

En cuanto a los medios municipales, Negre resalta la importancia de los boletines impresos y de las emisoras de radio locales. Sin embargo, considera que los boletines "nunca conseguirán efectos demasiado relevantes" (Ibid: 11) por las dificultades técnicas y por la limitación a la temática municipal. Huertas Claveria (1980:20) también se refiere a los boletines municipales, insistiendo en la necesidad de contar con una infraestructura profesional y en que los contenidos han de ser "lógicamente sometidos a la fiscalización de la Permanente" para evitar una revisión interminable.

Por el contrario, Negre cree que las emisoras de FM son el medio más importante y que se tenían que salvar las limitaciones en las concesiones de autorizaciones que se 
estaban dando e instaba a los ayuntamientos a "emitir sin necesidad de autorización pues el Gobierno no puede conculcar los derechos constitucionales" (Op. Cit.: 12). Considera que esas emisoras han de informar transparentemente sobre la acción de gobierno y procurar el acceso de las entidades y asociaciones, incidiendo especialmente en el "hecho cultural" y en la normalización del uso de la lengua catalana (Ibid: 12).

También Joan Castelló, consideraba que la radio era el medio más importante para los ayuntamientos, tanto es así que se refiere a ella "no como un medio de comunicación social, sino como El medio de comunicación social" (Castelló, 1981: 7), ya que las emisoras de FM se encontraban en un momento de gran expansión y credibilidad creciente. Desde ese punto de partida apoyaba el nacimiento de emisoras municipales, al igual que Negre, incluso sin licencia, amparadas en las libertades garantizadas por la Constitución, pero reclamando a la vez profesionalidad y centrarse en una misión "socio-educativa, divulgativa y de servicio al ciudadano" (Castelló, 1981: 8).

El vídeo y el cine fueron abordados por Pere Ignasi Fages (1981) quien apuntó que su utilización como instrumentos de comunicación entre administración y administrados resultaba poco viable, a pesar de sus posibilidades, por los escasos recursos existentes y por la falta de formación al respecto. Por el contrario, González y Carvajal (1981) expusieron la positiva experiencia que habían desarrollado como miembros del Institut del Cinema Català, que había producido 61 películas de 12 minutos, en catalán, para el Ayuntamiento de Barcelona, que se proyectaban en las salas de cine de la ciudad. Los autores consideran al cine como un medio de persuasión que ofrece "una información directa y descentralizada, pensada desde el lugar de donde parte, y con la mentalidad de quien lo realiza"; además "es importante por cuanto representa de normalización lingüística y cultural" (González y Carvajal, 1981: 12). En su texto se hacen también referencias al candente tema de la implantación de canales de televisión autonómicos, para referirse a la posibilidad de redifundir las películas y a la necesidad de financiación conjunta entre municipios y administraciones autonómicas.

En la misma línea, Toni Garriga, miembro de la Comisión Gestora de RTV Cardedeu, explicó la incipiente y pionera experiencia de esa televisión municipal, la primera en Cataluña, financiada a través de participaciones que se ofrecieron a los ciudadanos e impulsada y gestionada por una asociación con la finalidad de "elevar el nivel cultural de los ciudadanos", avanzar en la "integración colectiva" y apoyar el establecimiento de medios de comunicación en la población (Garriga, 1981:14). El artículo es realmente optimista respecto al futuro de las televisiones locales, sobre las que dice que pueden llegar a ser el medio más importante para evitar la homogeneización cultural. También este autor apela a los principios constitucionales para solventar el vacío legal sobre su regulación. 
Incluso se publicó una ponencia técnica sobre la utilización del cartel, de Josep Maria Trias-Folch (1981), en que se le atribuyen tres grupos de funciones: identificación, información y persuasión.

\section{Conclusiones y discusión}

La importancia de las Jornadas de Información y Comunicación Municipal deviene del momento en que se realizaron y del abanico de personalidades de diferentes ámbitos que participaron, conocedores tanto de la situación política y social en que se producía la comunicación, como de las dificultades profesionales y técnicas. El análisis de los textos publicados acerca las preocupaciones que los diferentes colectivos implicados percibían como trascendentales y las ópticas desde las que se abordaban.

Aunque a las Jornadas asistieron profesionales y representantes de las administraciones de todo el estado, se celebraron en Barcelona, ciudad en que la entidad organizadora tenía también su sede, y los ponentes, mayoritariamente, trabajaban en Cataluña. No es extraño que la iniciativa surgiera en ese contexto, puesto que a las cuestiones generales para el conjunto del estado se sumaban con fuerza las reivindicaciones culturales, tras años de dictadura y prohibiciones.

Por otra parte, como hemos visto, muchos de los ponentes se manifestaron políticamente de izquierdas sin ambages, criticando la actitud del gobierno del Estado y de los municipios gobernados por la derecha, pero sin esconder las malas prácticas o deficiencias de los ayuntamientos de izquierdas. Aunque hay ponencias de carácter técnico, en las recogidas especialmente en los dos primeros números de CEUMT dedicados a las Jornadas, se hacen propuestas manifiesta o claramente dirigidas a los ayuntamientos gobernados por la izquierda, para procurar tanto la coherencia entre la ideología y la acción política como para garantizar el éxito de los partidos afines en las siguientes convocatorias electorales 0 , incluso, para conseguir una transformación social radical.

Los temas que aparecen con mayor frecuencia, y que podemos considerar eran preocupaciones generalizadas en cuanto a la política comunicativa de los gobiernos municipales, fueron la necesidad de informar de la gestión de forma transparente y la de encontrar mecanismos de participación de la ciudadanía. Estas dos cuestiones aparecen de forma reiterada, exponiéndose tanto su necesidad como los problemas detectados para su implantación real. Junto a estas, también se insistió en la reivindicación de unos medios profesionales, superando la precariedad y el voluntarismo existentes para cumplir con el deber de ofrecer información precisa y completa, sin olvidar la importancia de la comunicación directa. 
La radio era el medio de comunicación mejor valorado, por su proximidad, inmediatez y por las oportunidades de participación que parecía ofrecer. Algunos trabajos también se refieren a la posibilidad de que la televisión ocupase en el futuro un papel preponderante. En ese sentido, no solamente se habló de la posibilidad de contar con canales municipales, sino también, de las expectativas depositadas en los canales de televisión autonómicos, especialmente en el caso de Cataluña. Igualmente, es interesante ver como en ese momento empieza a debatirse sobre la conveniencia de contar con planes de comunicación y de imagen corporativa.

Los textos de Vázquez Montalbán y Moragas se apoyan en trabajos de gran impacto en el campo de los estudios en comunicación que entroncan con el clima social de la transición, favorable a los medios comunitarios de origen social. Esas mismas circunstancias alentaron la creación de medios que emitían sin contar con licencia, como en el caso de RTV Cardedeu o la multitud de radios, varias veces citadas. Ante la falta de regulaciones, la libertad de expresión recogida en la Constitución, entonces aún de reciente aprobación, era el marco referencial primordial ya que la falta de desarrollo normativo no podía ser un freno a los derechos reconocidos.

Los trabajos que hemos analizado, publicados en un momento política y socialmente trascendental, son fuentes imprescindibles que permiten comprender cómo se gestaron las estructuras comunicativas de los ayuntamientos democráticos y los trabajos más recientes evidencian el enquistamiento de los problemas relacionados con la independencia de los profesionales, la participación ciudadana o la transparencia (Corcoy, 2016; Manfredi, Corcoy y Herranz, 2017; Molina et al., 2017; Moreno, Molina y Simelio, 2017). El lenguaje político y técnico utilizado en los textos y los medios de comunicación que se tratan evidencian el paso del tiempo, pero los problemas y expectativas que se plantean siguen siendo las cuestiones centrales que hoy nos preocupan.

En este trabajo nos hemos basado en unas fuentes documentales que consideramos excepcionalmente valiosas. Sin embargo, cabe contrastar los resultados que ofrecemos con nuevas investigaciones basadas en otros documentos del mismo periodo. En este sentido remitimos al trabajo de Marcial Murciano (1982), que ofrece una amplia relación de documentos académicos, informes, comunicaciones, etc., sobre los medios de proximidad en Cataluña. Igualmente, es necesario estudiar documentos que aborden la cuestión desde otros territorios del estado, con los que contrastar los resultados de este trabajo ya que, como se ha señalado, la presencia de personalidades de la comunicación en Cataluña es claramente mayoritaria en la muestra analizada. 


\section{Referencias bibliográficas}

$A B C$ (Sevilla), 19-10-1980. № 24.042, “Flash", p. 24.

BERTOT, J.C., JAEGER, P.T. Y GRIMES, J.M. (2010): "Using ICTs to create a culture of transparency: E-government and social media as openness and anti-corruption tools for societies" en Government Information Quarterly, no 27(3), pp. 264-271.

CAMPO VIDAL, M. (1980): "Prensa escrita (Resumen de la ponencia)" en CEUMT. La revista municipal, Edició Catalunya, no 32 (noviembre 1980), p. 20-23.

CASTELLÓ ROVIRA, J. (1981): "Las radios municipales y locales [sic]. Síntesis de la ponencia" en CEUMT. La revista municipal, Edició Catalunya, núm. 35 (febrero 1981), p. 7-8.

CASTELLS, M. (2009): Comunicación y poder. Madrid, Alianza.

CATALINA, B.; GARCÍA, A. (2013): "Herramientas interactivas y participación ciudadana en los ayuntamientos españoles" en Ámbitos, 22, pp. 1-21, en http://institucional.us.es/ambitos/?p=335.

CAVADAS, M. J. (2012): “Política local y comunicación: las webs de los ayuntamientos europeos" en TecCom Studies, no 3, p. 27-35.

CEUMT. La revista municipal (Edición Cataluña) (1980a): n 32, noviembre 1980.

-: (1980b): no 33, diciembre 1980.

-: (1981): no 35, febrero 1981.

CORCOY, M. (2016): Comunicació i periodisme a les corporacions locals: de les primeres eleccions democràtiques al retiment de comptes. Permanències $i$ canvis a les notícies, del paper als recursos digitals. Tesis doctoral, defensa 23/11/2016, en https://www.tesisenred.net/handle/10803/400073.

CORCOY, M., CARRASCO, M. Y GÓMEZ, P. (2001): Les Corporacions locals i la informació pública (1979-2001). Bellaterra: Laboratori de Periodisme $i$ Comunicació per a la Ciutadania Plural, UAB, en http://labcompublica.info/recerques/corporacions-locals-2011.

COSTA, LL. (2009): La comunicació local. Barcelona, UOC.

COSTA, P.O., CORCOY, M. Y GÓMEZ, P.L. (2006): Els governs locals i la Comunicació Pública. Cens dels mitjans públics catalans de comunicación local. Bellaterra, Universitat Autònoma de Barcelona, En http://labcompublica.info/recerques/cens-de-mitjans-2006/. 
DESTOT, M. (1981): "La información municipal en Francia. La experiencia de Grenoble" en CEUMT. La revista municipal, Edición Cataluña, no 35 (febrero 1981), p. 5-7.

ENZENSBERGER, H.M. (1972): Elementos para una teoría de los medios de comunicación. Barcelona, Anagrama.

FAGES, P.I. (1981): "El video y el cine" en CEUMT. La revista municipal, Edición Cataluña, no 35 (febrero 1981), p. 12-13.

FIGUERES, J.M. (1983): “Els butlletins d’informació municipal, línea per a la informació democrática" en CEUMT. La revista municipal. Edición Cataluña, no 59 (febrero 1983), p. 2-20.

GARRIGA, T. (1981): "Una tercera vía a la televisión. La experiencia de RTV Cardedeu" en CEUMT. La revista municipal, Edición Cataluña, no 35 (febrero 1981), p. 14-15.

GÉRTRUdiX, M., GERTRUdIS CASADO, M.C. Y ÁlVAREZ GARCíA, S. (2016): "Consumption of public institutions' open data by Spanish citizens" en El profesional de la información, no 25 (4), p. 535-544.

GONZÁLEZ, J.A. Y CARVAJAL, R. (1981): “El cine como soporte y la exhibición y distribución comercial como canal" en CEUMT. La revista municipal. Edición Cataluña, no 35 (febrero 1981), p. 5-7.

GUILLAMET, J. (1983): La premsa comarcal. Un model català de periodisme popular. Barcelona, Departament de Cultura de la Generalitat de Catalunya.

GUIMERÀ I ORTS, J. A. (2004): La comunicació local com a escenari de la diversitat cultural: el cas de Catalunya. Barcelona, Forum Universal de les Cultures, en http://www.portalcomunicacion.com/dialeg/paper/pdf/154_guimera.pdf.

GUIMERÀ I ORTS, J. A. Y ALBORCH GIL, F. (2011): "La crisis de la TDT local pública en España: el caso de Cataluña" en Revista Latina de Comunicación Social, no. 66, p. 292-312, en DOI: 10.4185/RLCS-66-2011-934-292-313.

HUERTAS CLAVERÍA, J.M. (1980): "Las oficinas de prensa y los boletines municipales" en CEUMT. La revista municipal. Edición Cataluña, no 32 (noviembre 1980), p. 1520.

JESSOP, B. (2003): "Governance and metagovernance: on reflexivity, requisite variety, and requisite irony" en BANG, H. (ed.). (2003). Governance, Governmentality, and Democracy. Manchester, Manchester University Press.

LA VANGUARDIA (1980a), 16-10-1980. № 35.563, “Actos para hoy", p.10.

- (1980b), 17-10-1980. N. 35.564, “Jornadas de información municipal”, p. 27. 
- (1980c), 18/10/1980. N. 35.565, “Departamentos políticos para información municipal", p. 26.

MACBRIDE, S. (et al.) (1980): Many voices, one world: communication and society today and tomorrow. London, Kogan.

MANFREDI SÁNCHEZ, J. L., CORCOY RIUS, M. Y HERRANZ DE LA CASA, J. M. (2017): “¿Noticias de alcance? El criterio periodístico en la publicación de noticias en las webs municipales españolas (2011-2016)" en El profesional de la información, v. 26, n. 3, pp. 412-420, en https://doi.org/10.3145/epi.2017.may.07.

MARISTANY, G.; MUSONS, A. (2002): Del desencant a la contrainformació. La premsa de barris a Barcelona. 1976-2001. Barcelona: Diputació de Barcelona, Col-legi de Periodistes de Catalunya.

MARTÍNEZ, M.; ROSENDE, S. (2011): “Participación ciudadana en las agendas 21 locales: cuestiones críticas de la gobernanza urbana" en Scripta Nova. Revista Electrónica de Geografía y Ciencias Sociales, vol. XV, no 355, en http://www.ub.es/geocrit/sn/sn-355.htm.

MOLINA RODRÍGUEZ-NAVAS, P. (2011): “Medios de comunicación local y democracia en Cataluña: estudios, propuestas e innovaciones" en Questión. Revista Especializada en Periodismo y Comunicación, vol. I, no 31, p. 1-14, en http://perio.unlp.edu.ar/ojs/index.php/question/article/view/1214.

MOLINA RODRÍGUEZ-NAVAS, P.; RODRÍGUEZ BREIJO, V.; CORCOY RIUS, M.; VADILLO BENGOA, N. (2017): "La transparencia de los ayuntamientos de Cataluña antes de la entrada en vigor de la legislación estatal y autonómica" en Anales de Documentación, $20 \quad$ (2), pp. 1-15, en http://dx.doi.org/10.6018/analesdoc.20.2.288151.

MONTALVO, F. (1980): "Las técnicas de la comunicación y la información" en CEUMT. La revista municipal. Edición Cataluña, no 33 (diciembre 1980), p. 19-23.

MORAGAS I SPA, M. (1980): “Democracia y políticas de comunicación. En el flujo internacional y la comunicación participativa" en CEUMT. La revista municipal. Edición Cataluña, no 33 (diciembre 1980), p. 9-15.

MORENO SARDÀ, A., MOLINA RODRÍGUEZ-NAVAS, P., CORCOY RIUS, M., AGUILAR PÉREZ, A., BORRÀS FARRAN, M. (2013): Infoparticip@: periodismo para la participación ciudadana en el control democrático. Criterios, metodologías y herramientas en Estudios sobre el mensaje periodístico, v. 19, n. 2, pp. 783-803, en https://ddd.uab.cat/pub/artpub/2013/129284/estsobmen_ a2013v19n2p783.pdf 
MORENO SARDÀ A.; MOLINA RODRÍGUEZ-NAVAS, P.; SIMELIO SOLÀ, N. (2017): "EI impacto de la legislación sobre transparencia en la información publicada por las administraciones locales" en El profesional de la información, vol. 26, n으 2, en https://recyt.fecyt.es/index.php/EPI/article/viewFile/epi.2017.may.03/35770.

MURCIANO, Marcial (1982): "Principals fonts documentals sobre els mitjans de comunicació a Catalunya (1976-1981)" en Anàlisi. Quaderns de Comunicació i Cultura, 6, pp. 91-120, en http://ddd.uab.cat/record/33290.

NEGRE i RIGOL, J. (1980): "Política de comunicación de los Ayuntamientos" en CEUMT. La revista municipal. Edición Cataluña, №. 32 (noviembre 1980), p. 9-12.

PETERS, B.G. (2000): “Governance and Comparative Politics" en PIERRE, J. (ed.). (2000). Debating Governance: Authority, Steering, and Democracy. Oxford, Oxford University Press, p. 36-53.

RIVERO MENÉNDEZ, J. A.; MORA AGUDO, L.; FLORES UREBA, S. (2007): “Un estudio de la rendición de cuentas a través del e-gobierno en la administración local española", en Empresa global y mercados locales: XXI Congreso Anual AEDEM, en https://dialnet.unirioja.es/descarga/articulo/2521491.pdf

ROJO, A (2005): "La gobernanza: un modelo alternativo de gestionar el conflicto" en Revista de pensamento do Eixo Atlântico, n요 8, p. 5-30.

ROMERO, J. Y FARINÓS, J. (2011): “Redescubriendo la gobernanza más allá del buen gobierno. Democracia como base, desarrollo territorial como resultado" en Boletín de la Asociación de Geógrafos Españoles, no 56, p. 295-319, en http://www.boletinage.com/articulos/56/13\%20AGE\%2056.pdf.

SEGURA, A. Y CARTES, F. (1980): “Campañas de concienciación”, en CEUMT. La revista municipal. Edición Cataluña, no 33 (diciembre 1980), p. 23-25.

SORIA, J.M. (1980). "Prensa y política municipal" en CEUMT. La revista municipal. Edición Cataluña, no 32 (noviembre 1980), p. 13-15.

TRIAS-FOLCH, J.M. (1981): "El cartel y sus funciones. Síntesis de ponencia" en CEUMT. La revista municipal. Edición Cataluña, no. 35 (febrero 1981), p. 15-17.

VÁZQUEZ MONTALBÁN, M. (1980): “Por una política comunicacional de masas" en CEUMT. La revista municipal. Edición Cataluña, no 33 (diciembre 1980), p. 16-19. 